\title{
Endothelial a6ß4 integrin protects during experimental autoimmune encephalomyelitis-induced neuroinflammation by maintaining vascular integrity and tight junction protein expression
}

Jennifer V. Welser, Sebok K. Halder, Ravi Kant, Amin Boroujerdi and Richard Milner*

\begin{abstract}
Background: Extracellular matrix (ECM) proteins play critical functions regulating vascular formation and function. Laminin is a major component of the vascular basal lamina, and transgenic mice deficient in astrocyte or pericyte laminin show defective blood-brain barrier (BBB) integrity, indicating an important instructive role for laminin in cerebral blood vessels. As previous work shows that in the normal brain, vascular expression of the laminin receptor a6ß4 integrin is predominantly restricted to arterioles, but induced on all vessels during neuroinflammation, it is important to define the role of this integrin in the maintenance of BBB integrity.

Methods: a6ß4 integrin expression was analyzed using dual immunofluorescence (dual-IF) of brain sections taken from the mouse model of multiple sclerosis, experimental autoimmune encephalomyelitis (EAE). To investigate the role of endothelial a6 $\beta 4$ integrin, transgenic mice lacking $\beta 4$ integrin in endothelial cells ( $\beta 4-E C-K O)$ and wild-type (WT) littermates were subject to EAE, and clinical score and various neuropathological parameters were examined by immunofluorescence. In addition, $\beta 4$ integrin null brain endothelial cells (BECs) were examined in culture for expression of tight junction proteins using immunocytochemistry and flow cytometry.

Results: Cerebrovascular expression of $\beta 4$ integrin was markedly upregulated during EAE progression, such that by the acute stage of EAE (day 21), the vast majority of blood vessels expressed $\beta 4$ integrin. In the EAE model, while the $\beta 4-E C-K O$ mice showed the same time of disease onset as the WT littermates, they developed significantly worse clinical disease over time, resulting in increased clinical score at the peak of disease and maintained elevated thereafter. Consistent with this, the $\beta 4-E C-K O$ mice showed enhanced levels of leukocyte infiltration and BBB breakdown and also displayed increased loss of the endothelial tight junction proteins claudin-5 and ZO-1. Under pro-inflammatory conditions, primary cultures of $\beta 4 \mathrm{KO}$ BECS also showed increased loss of claudin-5 and ZO-1 expression.
\end{abstract}

Conclusions: Taken together, our data suggest that a6 $\beta 4$ integrin upregulation is an inducible protective mechanism that stabilizes the BBB during neuroinflammatory conditions.

Keywords: Endothelial, Extracellular matrix, Laminin, Integrin, Blood-brain barrier, Vascular

\footnotetext{
* Correspondence: rmilner@scripps.edu

Department of Molecular Medicine, The Scripps Research Institute, 10550

North Torrey Pines Road, MEM-132, La Jolla, CA 92037, USA
} 


\section{Background}

Multiple sclerosis (MS) is a chronic inflammatory disease resulting in demyelination and degeneration of axons in the central nervous system (CNS) $[1,2]$. While the precise trigger of MS remains elusive, it is characterized pathologically by multiple inflammatory lesions of white matter that are separated in time and space. Though most of the damage is caused by infiltrating leukocytes, strong evidence suggests that alterations in vascular properties at an early stage of disease onset play a central role in the initiation and/or maintenance of this pathology by permitting inflammatory leukocytes to enter the CNS [3-6]. Blood vessels in the CNS are unique in forming the blood-brain barrier (BBB), which confers high electrical resistance and low permeability properties, thus protecting neural cells from potentially harmful blood components [7-11]. The molecular basis of the BBB depends on (i) inter-endothelial tight junction proteins, (ii) endothelial adhesion to the extracellular matrix (ECM) proteins of the underlying vascular basal lamina, and (iii) the influence of astrocyte end-feet and pericytes [11-14].

ECM proteins play critical functions shaping vascular formation during development and in the adult [15-19], making it a high priority to define the role of specific ECM proteins and their integrin receptors in regulating vascular remodeling and BBB integrity. The laminin receptor, $\alpha 6 \beta 4$ integrin, warrants special attention for several reasons. First, laminin is a major component of the basal lamina of blood vessels, and transgenic mice deficient in astrocyte or pericyte laminin show defective BBB integrity $[20,21]$. Second, in contrast to other laminin receptors such as $\alpha 6 \beta 1$ integrin and dystroglycan, in the normal brain, $\alpha 6 \beta 4$ integrin shows a limited distribution pattern, with expression restricted to arterioles [22]. Third, previous studies show that the number of cerebral vessels expressing $\alpha 6 \beta 4$ integrin is strongly increased in two models of neuroinflammation (GFAP-IL6 transgenic mice and a cerebral aneurysm model) [23, 24]. Fourth, in skin, $\alpha 6 \beta 4$ integrin plays a critical role in promoting mechanical stability and barrier integrity [25-27]. Taken together, these data raise the possibility that $\alpha 6 \beta 4$ integrin may play a protective role in the regulation of $\mathrm{BBB}$ integrity.

Previous studies have suggested a role for the $\beta 1$ class of integrins in modulation of BBB integrity [14], but so far, no one has defined a function for any one specific integrin. In light of the dynamic regulation of $\alpha 6 \beta 4$ integrin expression on cerebral blood vessels during neuroinflammation [23, 24], and its essential role in the maintenance of epidermal integrity in skin [25-27], the goal of this study was to examine the contribution of $\alpha 6 \beta 4$ integrin to $\mathrm{BBB}$ integrity by answering three main questions. First, how is endothelial $\beta 4$ integrin expression regulated in the mouse model of MS, experimental autoimmune encephalomyelitis (EAE)? Second, how does genetic deletion of endothelial $\beta 4$ integrin (using transgenic $\beta 4$-EC-KO mice) impact progression of EAE and its underlying pathology? Third, how does absence of endothelial $\beta 4$ integrin affect BBB integrity and endothelial expression of tight junction proteins?

\section{Methods}

\section{Animals}

The studies described have been reviewed and approved by The Scripps Research Institute Institutional Animal Care and Use Committee. The $\beta 4$ integrin $^{\text {flox/flox }}$ transgenic mice were a kind gift from Dr. Laura Feltri (University of Buffalo). The generation of Tie2-Cre, nestinCre, and $\beta 4$ integrin $^{\text {flox/flox }}\left(\beta 4\right.$ integrin $^{\mathrm{f} / \mathrm{f}}$ ) strains of mice and genotyping protocols have all been described previously [28-30]. All strains were backcrossed $>10$ times onto the $\mathrm{C} 57 \mathrm{BL} / 6$ background and maintained under specific pathogen-free conditions in the closed breeding colony of The Scripps Research Institute (TSRI).

\section{Experimental autoimmune encephalomyelitis (EAE)}

EAE was performed using a protocol and materials provided by Hooke Laboratories (Lawrence, MA). Briefly, 810 -week-old $\beta 4$-EC-KO or WT littermate control ( $\left.\beta 4^{\text {flox/wt }}\right)$ female mice were immunized subcutaneously with $100 \mu \mathrm{l}$ of $1 \mathrm{mg} / \mathrm{ml} \mathrm{MOG}_{33-35}$ peptide emulsified in complete Freud's adjuvant (CFA) containing $2 \mathrm{mg} / \mathrm{ml}$ Mycobacterium tuberculosis in both the base of the tail and upper back. In addition, on days 0 and 1 , the mice also received an intraperitoneal injection of $200 \mathrm{ng}$ pertussis toxin. The control mice received CFA containing no MOG peptide. This protocol leads to robust induction of clinical EAE on days 12-14 following immunization [31, 32]. The animals were monitored daily for clinical signs and scored as follows: 0-no symptoms, 1-flaccid tail, 2-paresis of hind limbs, 3-paralysis of hind limbs, 4-quadriplegia, and 5-death. The control and EAE mice were euthanized at different time points of disease, including $0,4,7,14,21$, and 35 days. Most histological studies were performed on tissue obtained at the 21-day time point, corresponding to the acute symptomatic stage of disease.

\section{Immunohistochemistry and antibodies}

Immunohistochemistry was performed on $10-\mu \mathrm{m}$ frozen sections of cold phosphate buffer saline (PBS)-perfused tissues as described previously [33]. Antibodies reactive for the following antigens were used in this study: rat monoclonals reactive to CD31 (MEC13.3), $\beta 4$ integrin (346-11A), MHC class II (M5/114.15.2), CD45, Mac-1 (M1/70), all from BD Pharmingen (La Jolla, CA); mouse monoclonal $\alpha$ SMA-Cy3 conjugate (1A4) from Sigma (St. Louis, MO); rabbit polyclonals reactive to claudin- 5 and ZO- 1 from Invitrogen (Carlsbad, CA); and fibrinogen from Millipore 
(Temecula, CA). Secondary antibodies used included Cy3conjugated anti-rat and anti-rabbit from Jackson Immunoresearch (West Grove, PA) and anti-rat Alexa Fluor 488 and anti-rabbit Alexa Fluor 568 from Invitrogen (Carlsbad, CA).

\section{Image analysis}

Images were taken using a $\times 20$ objective on a Zeiss Imager M1.m. microscope. Analysis was performed specifically in the medulla oblongata region of the brain. For each antigen, four images were taken per region at $\times 20$ magnification, and a minimum of three sections per brain were analyzed to calculate the mean for each subject. All data analysis was performed using NIH ImageJ software. This analysis was performed using four animals of each genotype per condition per experiment, and the results were expressed as the mean \pm SEM. Statistical significance was assessed by using the Student's $t$ test, in which $p<0.05$ was defined as statistically significant. Blood-brain barrier (BBB) integrity was evaluated by measuring extravascular leakage of fibrinogen, in which the total area of fibrinogen staining per field of view (FOV) was divided by the total vascular area (CD31 signal) in the same FOV. Vascular expression of tight junction proteins was evaluated by measuring the total claudin- 5 or ZO- 1 area per FOV divided by the total vascular area for the same FOV.

\section{Cell culture}

Pure cultures of primary mouse brain endothelial cells (BECs) derived from the $\beta 4-\mathrm{EC}-\mathrm{KO}$ or littermate control mice were prepared as previously described [34, 35]. Briefly, the brains were removed from the 8-week-old mice, minced, dissociated for $1 \mathrm{~h}$ in papain and DNase I, and centrifuged through $22 \%$ BSA to remove myelin, and endothelial cells were cultured in endothelial cell growth media (ECGM) consisting of Hams F12, supplemented with 10\% FBS, heparin, ascorbic acid, L-glutamine, penicillin/streptomycin (all from Sigma), and endothelial cell growth supplement (ECGS) (Upstate Cell Signaling Solutions, Lake Placid, NY), on type I collagen (Sigma)-coated six-well plates. To obtain BECs, puromycin $(4 \mu \mathrm{g} / \mathrm{ml}$, Alexis $\mathrm{GmbH}$, Grunberg, Germany) was included in culture media between days 1-3 to remove contaminating cell types. Endothelial cell purity was $>99 \%$ as determined by CD31 in flow cytometry. For all experiments, BECs were used only for the first passage.

\section{Immunofluorescence of primary BECs}

Coverslips were coated with laminin (Sigma, $10 \mu \mathrm{g} / \mathrm{ml}$ ) for $2 \mathrm{~h}$ at $37^{\circ} \mathrm{C}$, and BECs from the $\beta 4$-EC-KO or wild-type littermate mice were plated onto them. Upon reaching confluence, the cells were treated with either $10 \mathrm{ng} / \mathrm{ml}$ TNF- $\alpha$ or IFN- $\gamma$ (both from R\&D, Minneapolis, MN) for $48 \mathrm{~h}$. After this time, the cells were fixed for $5 \mathrm{~min}$ in acetone/methanol (1:1) at $-20{ }^{\circ} \mathrm{C}$ and blocked for $30 \mathrm{~min}$ in
5\% normal goat serum in PBS containing 0.2\% triton X100 (Sigma) to permeabilize the cells (maintained in all incubation steps thereafter). The cells were then incubated with a rabbit polyclonal anti-claudin-5 antibody (Invitrogen) for $1 \mathrm{~h}$ followed by anti-rabbit-Cy3 secondary (Jackson Immunoresearch) for $1 \mathrm{~h}$, labeled with the nuclear marker Hoechst (Sigma) for 5 min before being washed and mounted on glass slides.

\section{Flow cytometry}

Brain endothelial cell (BEC) expression of claudin-5 and ZO-1 was examined as described previously [36]. Briefly, BECs derived from the $\beta 4-\mathrm{EC}-\mathrm{KO}$ or littermate control mice were first passaged onto laminin-coated six-well plates. Upon reaching confluence, the cells were treated with either $10 \mathrm{ng} / \mathrm{ml}$ TNF- $\alpha$ or IFN- $\gamma$ (both from R\&D) for $48 \mathrm{~h}$ and then removed and cellular expression of claudin-5 and ZO-1 analyzed by flow cytometry using rabbit polyclonal anti-claudin-5 or anti-ZO-1 antibodies followed by anti-rabbit Alex Fluor 488 secondary (both from Invitrogen). As the claudin-5 and ZO-1 antibodies are directed against intracellular epitopes, the cells were first fixed and then permeabilized using the Cytofix/ Cytoperm kit (BD Pharmingen) and all subsequent incubations were performed in the cytoperm buffer. The fluorescent intensity of labeled cells was analyzed with a Becton Dickinson FACScan machine, with 10,000 events captured for each condition. Each experiment was repeated a minimum of four times and the data expressed as mean \pm SEM fluorescent intensity. Statistical significance was assessed by using the Student's paired $t$ test, in which $p<0.05$ was defined as statistically significant.

\section{Results}

B4 integrin is strongly upregulated on brain endothelial cells during neuroinflammation in the EAE model

Extracellular matrix (ECM) proteins play critical functions influencing vascular cell behavior both during development and in the adult [15-19]. In light of the key role of laminin in contributing to blood-brain barrier (BBB) integrity of cerebral blood vessels [20, 21], it is a high priority to define the role of specific integrin laminin receptors in mediating these effects. The bestdefined laminin receptors are the $\alpha 6$ integrins, $\alpha 6 \beta 1$ and $\alpha 6 \beta 4$, functional heterodimers comprising the $\alpha 6$ integrin subunit coupled with either the $\beta 1$ or $\beta 4$ integrin subunits, respectively [37]. Interestingly, in a previous study, we found that while the $\alpha 6$ and $\beta 1$ integrin subunits are expressed universally by all cerebral blood vessels, expression of the $\beta 4$ integrin subunit is restricted predominantly to endothelial cells within arterioles [22]. As shown in Fig. 1 (top row), dual immunofluorescence (dual-IF) with the endothelial marker CD31 shows that $\beta 4$ integrin is expressed on only a small fraction of 


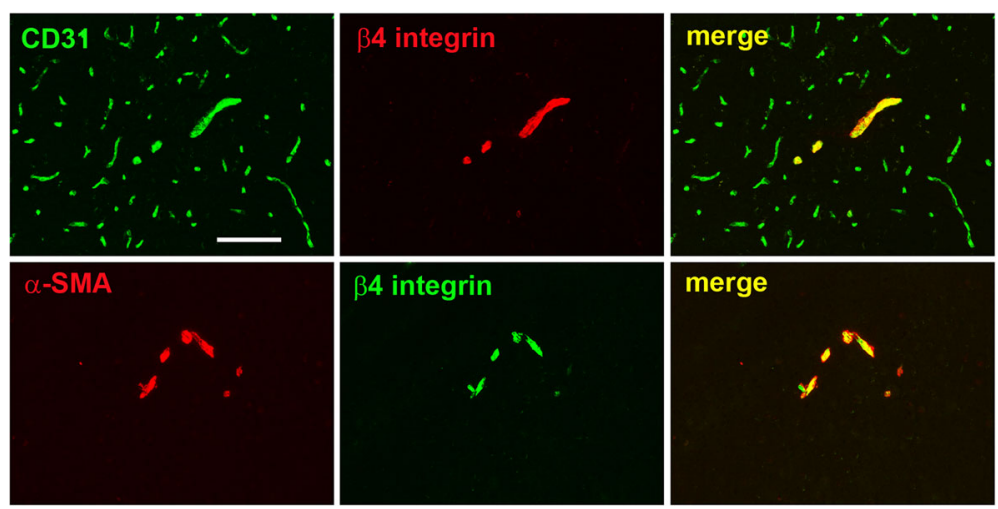

Fig. 1 Characterization of $\beta 4$ integrin expression on blood vessels in the normal brain. Top panel shows dual-IF on frozen sections of the medulla oblongata from adult mice using antibodies specific for the endothelial marker CD31 (AlexaFluor-488, green) and $\beta 4$ integrin (Cy3, red). Lower panel shows dual-IF using antibodies specific for smooth muscle cell marker a-SMA (Cy3, red) and $\beta 4$ integrin (AlexaFluor-488, green). Scale bar $=100 \mu \mathrm{m}$. Note that $\beta 4$ integrin was expressed by only a fraction of CD31-positive vessels, but co-localized strongly with a-SMA

cerebral blood vessels, which tend to be larger diameter vessels. The lower row of Fig. 1 reveals that $\beta 4$ integrin co-localizes strongly with $\alpha$-smooth muscle actin $(\alpha-$ SMA), a marker of arterial vessels, indicating that $\beta 4$ integrin is expressed predominantly in cerebral arterioles.

Previous studies have described strong upregulation of $\beta 4$ integrin on cerebral vessels in two inflammatory models: cerebral aneurysms and the GFAP-IL6 transgenic mouse [23, 24]. To determine whether cerebrovascular levels of $\beta 4$ integrin are also upregulated in neuroinflammatory demyelinating disease, we studied this process in the experimental autoimmune encephalomyelitis (EAE) mouse model of chronic progressive multiple sclerosis (MS). EAE was induced in 8-10-week-old female $\mathrm{C} 57 \mathrm{Bl} / 6$ mice by immunization with $\mathrm{MOG}_{35-55}$ peptide, as previously described [38]. In keeping with findings from our lab and others $[31,32,38]$, the mice began developing clinical signs 12 days postimmunization and the disease gradually got more severe over time. Clinical severity peaked around 21 days (acute symptomatic phase), and improved slightly thereafter, but never completely recovered at the experimental endpoint of 35 days (chronic symptomatic phase) (Fig. 2a). In this model, robust inflammation is observed in the hindbrain, particularly in the medulla oblongata and cerebellum areas $[31,32,38]$. To examine whether $\beta 4$ integrin expression levels are regulated during EAE progression, we performed CD31//4 integrin dual-IF staining on frozen brain sections at $0,4,7,14,21$, and 35 days post-immunization. As shown in Fig. 2b, c, under control conditions (day 0), $\beta 4$ integrin was expressed by only a small fraction of cerebral blood vessels in the medulla region of the brain, but as EAE developed, an increasing number of blood vessels showed expression of the $\beta 4$ integrin subunit, such that by the acute stage of EAE (day 21), the vast majority of blood vessels expressed $\beta 4$ integrin, and this expression was maintained high through to the chronic stage (day 35). Quantification revealed that compared to control (diseasefree) conditions, significant upregulation of endothelial $\beta 4$ integrin was first detected 7 days post-immunization $(9.9 \pm 2.1$ compared to $5.7 \pm 0.8 \beta 4$ integrin + vessels per field of view (FOV) $p<0.05$ ); this reached a peak after 21 days ( $34.3 \pm 4.4$ compared to $5.7 \pm 0.8 \beta 4$ integrin + vessels per FOV, $p<0.01$ ) and was still maintained 35 days post-immunization $(32.5 \pm 3.9$ compared to $5.7 \pm 0.8 \beta 4$ integrin + vessels per FOV, $p<0.01$ (Fig. 2b).

\section{Genetic deletion of endothelial $\beta 4$ integrin results in worse clinical disease and neuroinflammation in EAE}

Previous work has shown that $\beta 4$ integrin plays an essential protective role in the skin by promoting mechanical and barrier integrity in epidermal cells, such that $\beta 4$ integrin global $\mathrm{KO}$ mice show a perinatal lethal phenotype as a result of epidermal detachment and severe skin blistering [25-27]. To examine whether absence of endothelial $\beta 4$ integrin has any functional consequences on the vascular integrity of cerebral blood vessels, we used a Cre-Lox approach to generate mice lacking $\beta 4$ integrin specifically in endothelial cells ( $\beta 4-\mathrm{EC}-\mathrm{KO}$ strain), by crossing floxed $\beta 4$ integrin mice with Tie2-Cre transgenic mice, as previously described [22]. The $\beta 4-\mathrm{EC}-\mathrm{KO}$ mice are viable and fertile and show no obvious defects in developmental angiogenesis or vascular function in the adult [22] and thus are amenable to experimental analysis. EAE was induced in the 8-10-week-old female $\beta 4$-EC-KO and WT littermate mice and disease progression compared (Fig. 3). This showed that while the $\beta 4$-EC-KO mice showed the same time of onset of EAE as the WT littermates, over time, they developed significantly worse clinical disease, resulting in an increased mean clinical score at the peak of disease, and this elevated mean clinical score was maintained all the way 


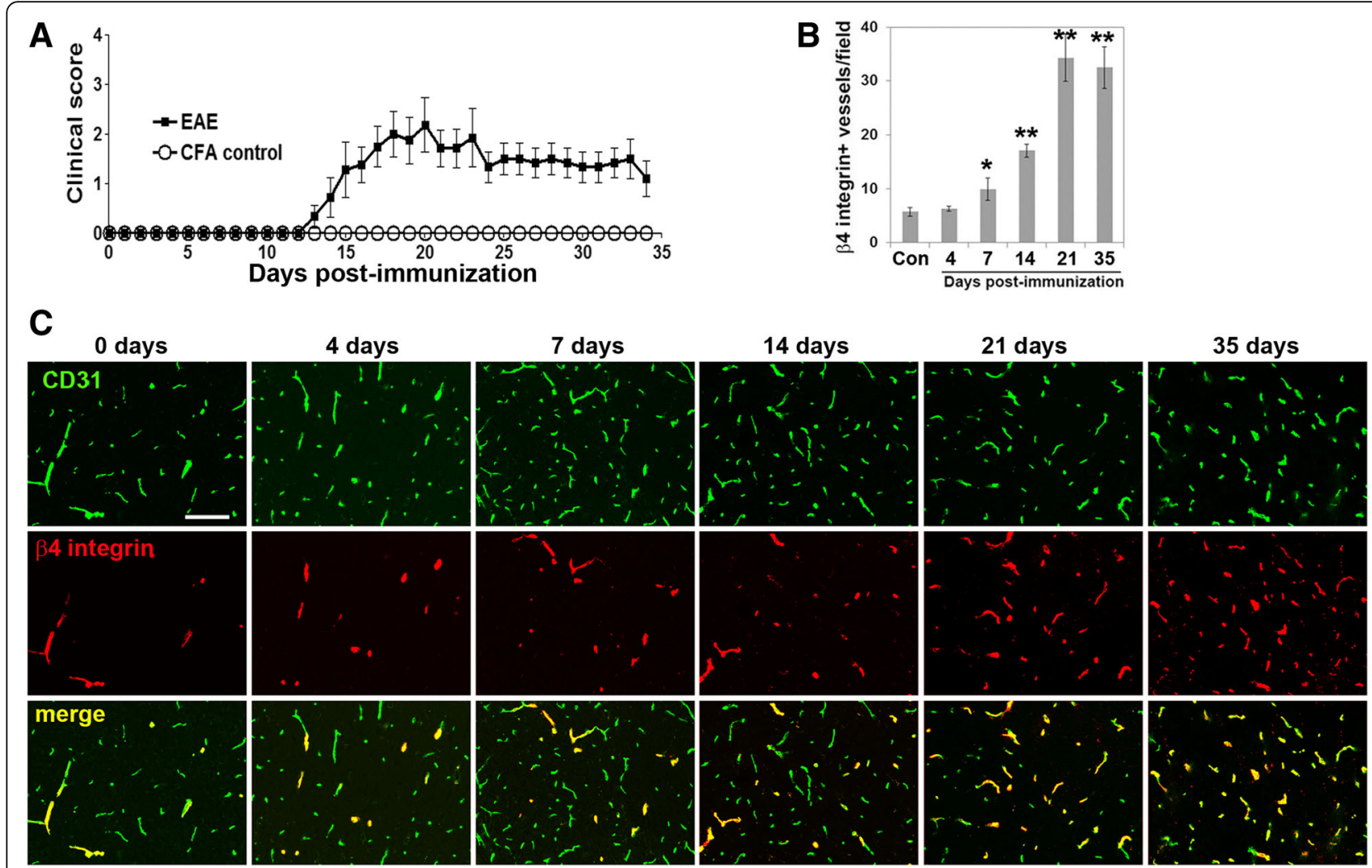

Fig. 2 Vascular $\beta 4$ integrin expression is strongly upregulated during the development of EAE. a Time course of increasing EAE severity (clinical score) with time post-immunization. $\mathbf{b}$ Quantification of the number of blood vessels expressing $\beta 4$ integrin in frozen sections of the medulla oblongata during EAE progression. Note that significant upregulation of endothelial $\beta 4$ integrin was detected 7 days post-immunization and peaked after 21 days. ${ }^{*} p<0.05,{ }^{* *} p<0.01$. c Frozen sections of the medulla oblongata at different time points of EAE progression were dualstained using antibodies specific for the endothelial marker CD31 (AlexaFluor-488, green) and $\beta 4$ integrin (Cy3, red). Scale bar $=100 \mu \mathrm{m}$. Note that in the disease-free brain (0 days), $\beta 4$ integrin was expressed by only a fraction of CD31-positive vessels, but the number of vessels expressing $\beta 4$ integrin gradually increased with EAE progression, such that by the acute stage of EAE (day 21), the vast majority of blood vessels expressed $\beta 4$ integrin, and this expression was maintained high through to the chronic stage (day 35)

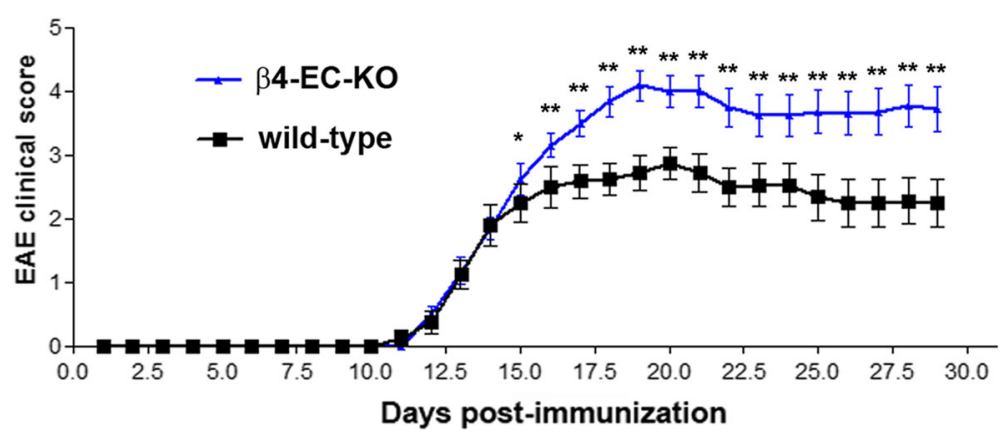

Fig. 3 The impact of genetically deleting endothelial $\beta 4$ integrin on clinical severity in EAE. The progression of EAE in the $\beta 4-E C-K O$ and WT littermate control mice was evaluated by measuring clinical score on daily intervals. All points represent the mean \pm SD ( $n=16$ mice per strain). Note that while the $\beta 4-E C-K O$ mice showed the same time of disease onset as the WT littermates, over time, they developed significantly worse clinical disease, resulting in increased mean clinical score for the duration of the experiment. This result was confirmed in three separate experiments. ${ }^{*} p<0.05,{ }^{* *} p<0.01$ 
from a relatively early stage of disease (day 15 postimmunization), through the acute stage (day 20-day 22) right up to day 30 (chronic stage) (Fig. 3). This result was confirmed in three separate experiments.

To investigate how lack of endothelial $\beta 4$ integrin affects neuroinflammation in the EAE model, we used IF to examine the distribution of three markers of inflammatory cells in the medulla region of the brain: MHC class II, CD45, and Mac-1. As shown in Fig. 4, quantification of all three markers at the acute phase of EAE (day 21) revealed that compared to the WT controls, $\beta 4$-EC-KO brains contained increased numbers of $\mathrm{MHC}$ II $+(60.3 \pm 6.8$ vs. $39.5 \pm 5.4$ MHC II+ cells per FOV, $p<0.05)$ and increased numbers of CD45+ inflammatory leukocytes $(67.8 \pm 1.2$ vs. $28.5 \pm 2.4$ CD $45+$ cells per FOV, $p<0.01)$, as well as increased expression level of the microglial/macrophage marker Mac-1 (62.9 \pm 1.1 vs. $41.1 \pm 5.7$ expression level per FOV, $p<0.05)$. Similar observations were also found in the cerebellum, another area showing marked leukocyte infiltration in the EAE model. Thus, in this EAE model, absence of endothelial $\beta 4$ integrin resulted in worse clinical disease, correlating with increased neuroinflammation.

In $E A E$, cerebral blood vessels in $\beta 4-E C-K O$ show enhanced vascular leakage, correlating with reduced expression of tight junction proteins

In light of the barrier-stabilizing role of $\alpha 6 \beta 4$ in the skin [25-27], and our data showing worse clinical disease and leukocyte infiltration in the EAE model, we next examined whether lack of endothelial $\beta 4$ integrin results in weakened vascular integrity of cerebral blood vessels. Using fibrinogen leakage as a marker of BBB breakdown, CD31/fibrinogen dual-IF of the medulla showed that in the majority of blood vessels, fibrinogen staining was localized to within the lumen of blood vessels (Fig. 5), consistent with previous findings [38]. However, in some blood vessels during the acute stage of EAE, fibrinogen staining also extended beyond the vascular margin, creating a fuzzy leakage pattern around some blood vessels, indicating vascular breakdown. Interestingly, the pattern of fibrinogen leakage appeared noticeably worse in the $\beta 4$ EC-KO mice compared to the WT controls, both in terms of the number of leaky vessels and the extent of leakage. Fibrinogen leakage was quantified using ImageJ analysis software and revealed that the extent of fibrinogen leakage was significantly greater in the $\beta 4-\mathrm{EC}-\mathrm{KO}$ mice compared to the WT littermate controls $(2.13 \pm 0.35$ compared to $1.27 \pm 0.06$ fibrinogen/CD31 ratio as described in the "Methods" section, $p<0.05$, Fig. 5b).

As cerebral blood vessels rely on expression of tight junction proteins such as claudin-5 and ZO-1 to maintain high vascular integrity $[7,9,11]$, we next examined whether expression of these proteins is altered in cerebral blood vessels of the $\beta 4-\mathrm{EC}-\mathrm{KO}$ mice during EAE by performing dual-IF of CD31/claudin-5 or CD31/ZO-1 on brain sections taken from the $\beta 4-\mathrm{EC}-\mathrm{KO}$ or WT
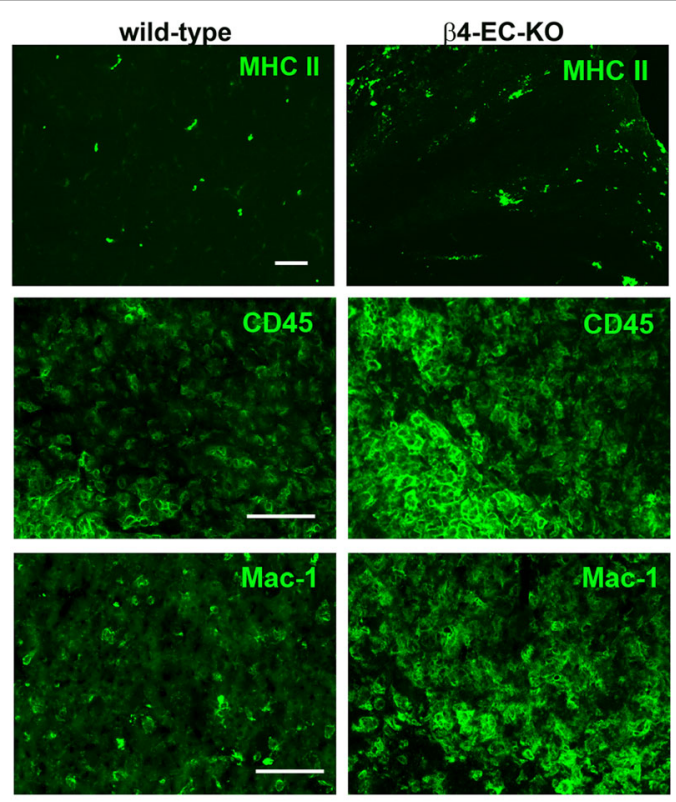

Fig. 4 The impact of genetically deleting endothelial $\beta 4$ integrin on neuroinflammation in EAE. Frozen brain sections taken from the $\beta 4$-EC-KO and WT littermate control mice at the acute stage of EAE (day 21) were stained using antibodies specific for the inflammatory leukocyte markers MHC II, CD45, and Mac-1. Data points represent the mean \pm SEM of events observed in the medulla oblongata $(n=4 \mathrm{mice})$. Scale bar $=100 \mu \mathrm{m}$. Note that quantification of all three markers revealed that $\beta 4-E C-K O$ brains contained increased numbers of MHC $I+$ and CD45+ inflammatory leukocytes, as well as increased expression level of the microglial/macrophage marker Mac-1 

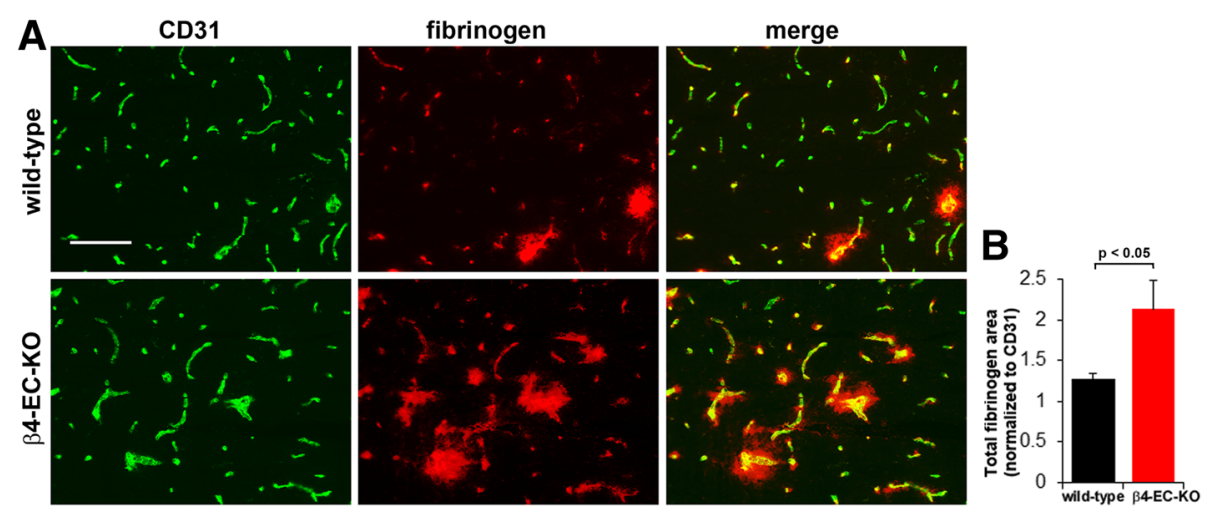

Fig. 5 Evaluating the impact of genetically deleting endothelial $\beta 4$ integrin on BBB integrity in EAE. a Frozen sections of the medulla oblongata taken from the $\beta 4-E C-K O$ and WT littermate control mice at the acute stage of EAE (day 21) were dual-stained using antibodies specific for the endothelial marker CD31 (AlexaFluor-488, green) and fibrinogen (Cy3, red). Scale bar $=100 \mu \mathrm{m}$. b Quantification of fibrinogen leakage in the $\beta 4-E C-K O$ vs. WT littermate mice. Data points represent the mean \pm SEM of events observed in the medulla oblongata ( $n=4$ mice). Scale bar $=100 \mu \mathrm{m}$. Note that that in most blood vessels, fibrinogen staining was localized to within the lumen of blood vessels. However, in some blood vessels, fibrinogen staining extended beyond the vascular margin, creating a fuzzy leakage pattern, indicative of loss of vascular integrity. Also note that in the $\beta 4-\mathrm{EC}-\mathrm{KO}$ mice, fibrinogen leakage was noticeably worse than the WT controls, both in the number and extent of leaky vessels, and this was confirmed by quantification

littermate mice during the acute stage of EAE. This showed that consistent with previous reports, claudin5 and ZO-1 co-localize very tightly with the endothelial cell marker CD31 on all blood vessels (Fig. 6). Importantly, no obvious difference in the expression level of claudin-5 or ZO-1 was detected between the $\beta 4-E C-K O$ or WT littermate mice under disease-free conditions or in the pre-symptomatic phase (14 days post-immunization) (Fig. 6c, d). However, during the acute phase of EAE (21 days post-immunization), the vascular expression levels of claudin-5 and ZO-1 were significantly reduced compared to the disease-free control levels, and interestingly at the acute phase of disease, levels in the $\beta 4$-EC-KO mice were markedly lower than those in the WT littermates. By quantifying the ratio of tight junction protein area to CD31 area (to allow for variations in vascular density between different FOVs), this revealed that in the WT mice, vascular claudin-5 levels were reduced from $0.92 \pm 0.06$ under control conditions to $0.69 \pm 0.12$ in the acute phase of $\operatorname{EAE}(p<0.05)$ and at this stage of EAE, claudin-5 levels in the $\beta 4-\mathrm{EC}-\mathrm{KO}$ mice were significantly lower than their WT littermates $(0.47 \pm 0.07$ vs. $0.69 \pm 0.12, p<0.05)$. In a similar manner, vascular expression of $\mathrm{ZO}-1$ in the brains of the WT mice was reduced from $0.77 \pm 0.05$ under control conditions to $0.33 \pm 0.06$ in the acute phase of $\operatorname{EAE}(p<0.05)$, and at this stage of EAE, ZO-1 levels in the $\beta 4$-EC-KO mice were significantly lower than their WT littermates $(0.24 \pm 0.01$ vs. $0.33 \pm 0.06, p<0.05)$. Thus, during the acute stage of EAE, cerebrovascular levels of both claudin- 5 and ZO-1 were significantly lower in the $\beta 4-E C-K O$ mice than their WT littermates.
Under pro-inflammatory conditions, $\beta 4$ integrin null BECs show reduced levels of claudin-5 and ZO- 1

Following on from our observation that in EAE-affected brain, blood vessels in the $\beta 4$-EC-KO mice appear to show greater loss of claudin-5 than the WT controls, we wanted to test directly whether absence of $\beta 4$ integrin impacts the expression of claudin-5 or ZO- 1 at cell-cell junctions. To examine this more closely at the cellular level, we isolated primary brain endothelial cells (BECs) from the $\beta 4-\mathrm{EC}-\mathrm{KO}$ and WT littermate mice and cultured them on laminin (the ECM ligand for $\alpha 6 \beta 4$ integrin) until confluent, at which point TNF- $\alpha$ was added to mimic inflammatory conditions. Forty hours later, claudin-5 expression was examined by immunocytochemistry. As shown in Fig. 7a, under control conditions, $\beta 4$ integrin $\mathrm{KO}$ and WT BECs expressed equivalent levels of claudin-5. However, after culture with TNF- $\alpha, \beta 4$ integrin KO BECs showed much weaker expression of claudin- 5 at cell-cell junctions. To confirm this result, we examined claudin- 5 expression by flow cytometry as previously described [14]. This showed that while ZO-1 expression in WT BECs was significantly reduced by TNF- $\alpha$ (from $776 \pm 59$ under control conditions to $546 \pm 46.1, p<0.05)$ and IFN- $\gamma$ (from $776 \pm 59$ under control conditions to $601 \pm 47.3, p<0.05$ ), expression of claudin -5 was not significantly affected by either of these cytokines. Interestingly, while $\beta 4$ integrin $\mathrm{KO}$ and WT BECs expressed equivalent levels of claudin-5 and ZO-1 under basal conditions, after exposure to TNF- $\alpha$ or IFN- $\gamma$, $\beta 4$ integrin KO cells expressed significantly lower levels of claudin- 5 (with TNF- $\alpha$ treatment, $454 \pm 63.1$ vs. $739 \pm 54.2, p<0.05$ and with IFN- $\gamma$ treatment, $532 \pm 49.3$ vs. $801 \pm 121.4, p<0.05$ ) (Fig. 7b) and ZO-1 (with TNF- $\alpha$ treatment, $357 \pm 49.8$ vs. 

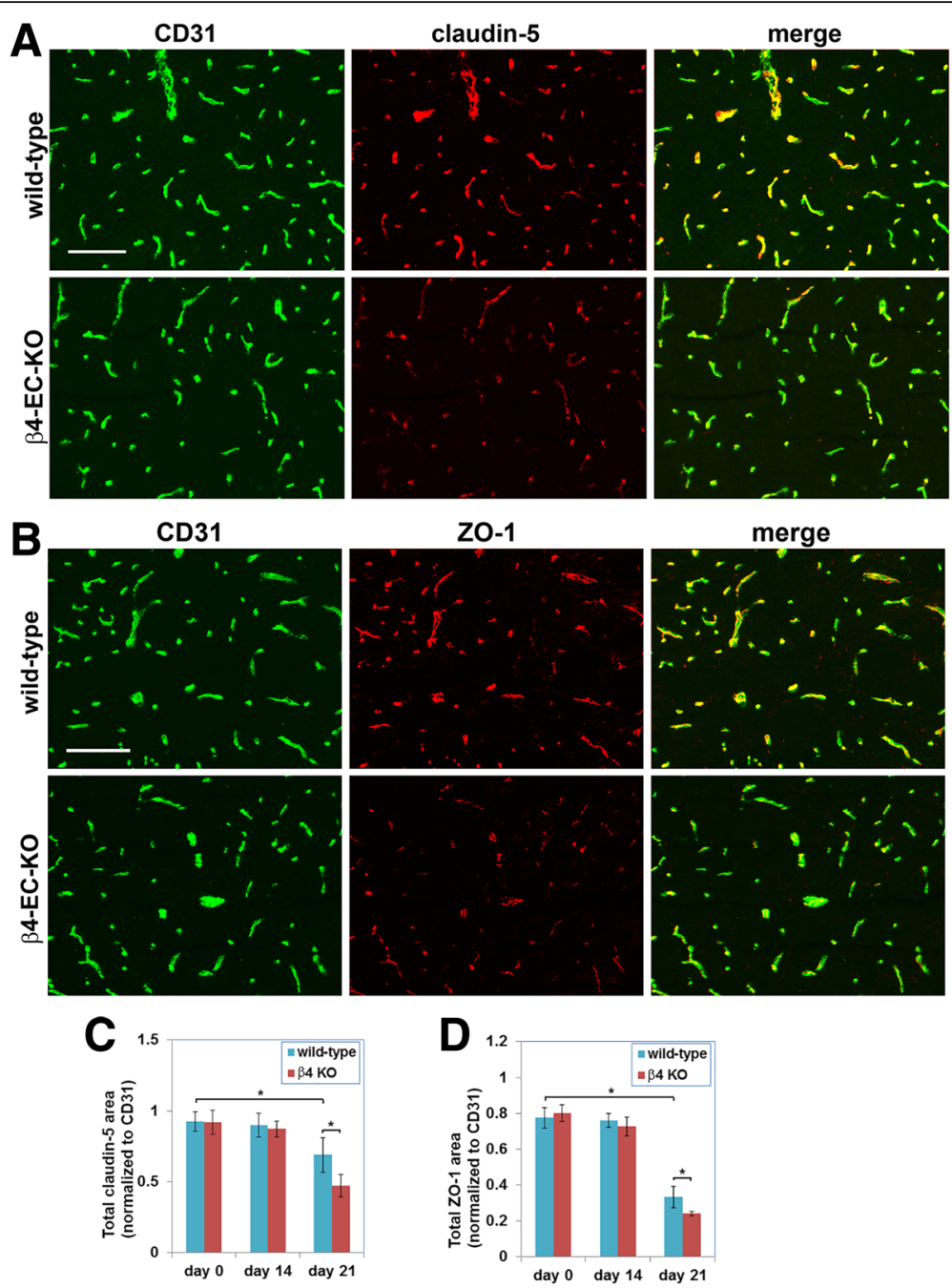

Fig. 6 Evaluating the impact of genetic deletion of endothelial $\beta 4$ integrin on endothelial tight junction protein expression in EAE. a, b Frozen sections of the medulla oblongata taken from the $\beta 4-E C-K O$ and WT littermate control mice at the acute stage of EAE (day 21) were dual-stained using antibodies specific for the endothelial marker CD31 (AlexaFluor-488, green) and claudin-5 (Cy3, red) or CD31 (AlexaFluor-488, green) and ZO-1 (Cy3, red) in A and B, respectively. Scale bar $=100 \mu \mathrm{m}$. c, d Quantification of endothelial expression of claudin-5 (c) and ZO-1 (d) in the $34-\mathrm{EC}-\mathrm{KO}$ vs. WT littermate mice. Data points represent the mean \pm SEM of events observed in the medulla oblongata ( $n=4$ mice). Note that in the WT mice, endothelial levels of claudin-5 and ZO-1 were markedly reduced in the acute phase of EAE (day 21), and at this phase of disease, expression levels in the $\beta 4$-EC-KO mice were significantly reduced compared to their WT littermates

$546 \pm 46.2, p<0.05$ and with IFN- $\gamma$ treatment, $404 \pm 25.5$ vs. $601 \pm 47.1, p<0.05$ ) (Fig. 7c). These in vitro results support our in vivo observations and suggest that $\beta 4$ integrin confers vasculo-protection in part by promoting vascular integrity via stabilization of the tight junction proteins claudin-5 and ZO-1.

\section{Discussion}

The goal of this study was to examine the contribution of endothelial $\alpha 6 \beta 4$ integrin to BBB integrity during neuroinflammatory disease. Previous studies have described marked upregulation of $\alpha 6 \beta 4$ integrin expression on cerebral blood vessels during neuroinflammation [23, 24]. Taken with its essential role in the maintenance of epidermal integrity in skin [25-27], this prompted us to wonder if $\alpha 6 \beta 4$ integrin induction on brain endothelium might be part of a physiological adaptive response in an attempt to secure BBB integrity under neuroinflammatory conditions. In this study, we set out to answer this question by using the mouse EAE model of chronic progressive MS. Our main findings were (i) $\alpha 6 \beta 4$ integrin is strongly upregulated on all cerebral blood vessels during the progression of EAE; (ii) genetic deletion of endothelial $\beta 4$ integrin ( $\beta 4$-EC-KO mice) results in worse clinical disease in $\mathrm{EAE}$, correlating with increased leukocyte infiltration into the CNS; (iii) during EAE, cerebral blood vessels in the $\beta 4$-EC-KO mice show enhanced vascular leakage of the plasma protein fibrinogen; (iv) this vascular leak 


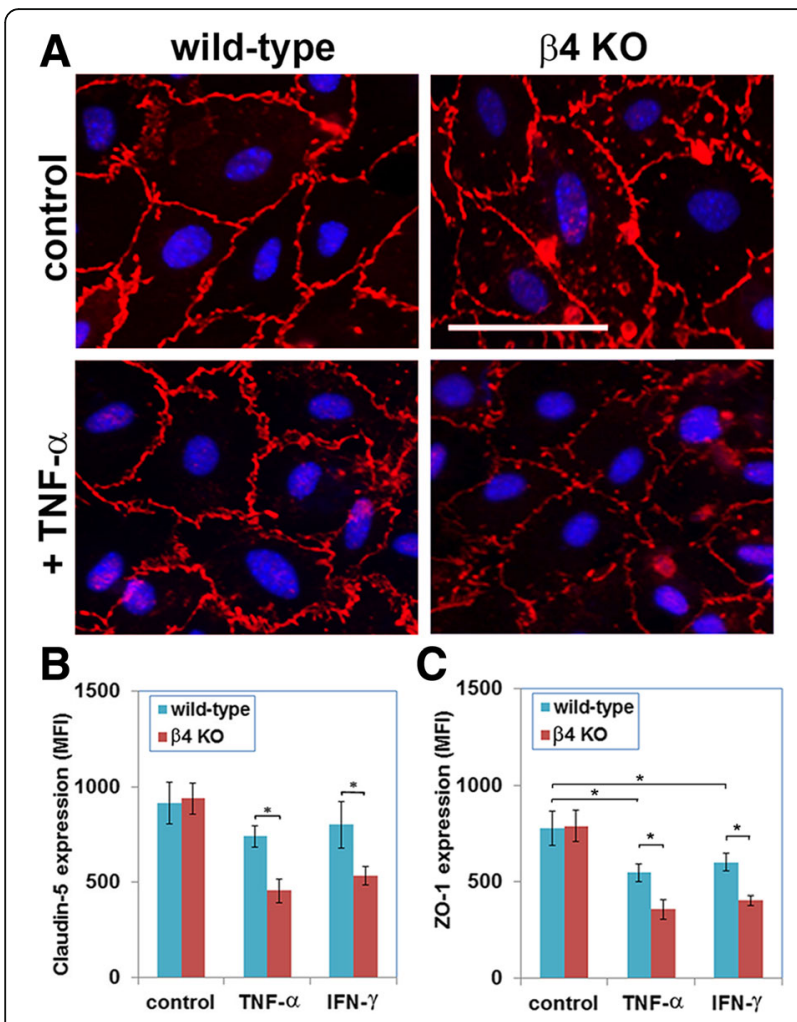

Fig. 7 Evaluating endothelial tight junction protein expression in primary brain endothelial cells (BECs). a BECs from the $\beta 4-E C-K O$ and WT littermate control mice were cultured on laminin in the presence or absence of TNF-a, and claudin-5 expression was examined by IF. b, c Quantification of claudin-5 (b) or ZO-1 (c) expression by $\beta 4$ integrin null and WT BECs under pro-inflammatory conditions (treatment with TNF-a or IFN- $\gamma$ ). All points represent the mean \pm SEM of the mean fluorescent intensity (MFI) of three separate experiments. Note that while ZO-1 expression in WT BECs was significantly reduced by TNF- $a$ and IFN- $\gamma$, expression of claudin-5 was not significantly affected by either of these cytokines. Furthermore, compared to WT BECs, while $\beta 4$ integrin $\mathrm{KO}$ cells expressed equivalent levels of claudin-5 and ZO-1 under basal conditions, after exposure to TNF- $a$ or IFN- $\gamma, \beta 4$ integrin KO BECs expressed significantly lower levels of claudin-5 and ZO-1. * $p<0.05$

correlates with reduced expression of the tight junction proteins claudin-5 and ZO-1 in the blood vessels of the $\beta 4-E C-K O$ mice; and (v) parallel in vitro studies showed that under pro-inflammatory conditions, primary cultures of $\beta 4 \mathrm{KO}$ BECs also showed reduced expression of claudin-5 and ZO-1 expression. Taken together, these studies support the notion that $\alpha 6 \beta 4$ integrin upregulation is an inducible protective mechanism that stabilizes the BBB during neuroinflammatory conditions.

The role of cell adhesion molecules in maintaining BBB integrity

Constituent cells of the BBB adhere to vascular basal lamina proteins such as laminin, collagen IV, fibronectin, and heparin sulfate proteoglycan (HSPG) predominantly via cell surface receptors of the integrin family [13, 37]. These interactions between ECM proteins and their cognate receptors regulate not just cell adhesion but also many aspects of cell behavior including survival, proliferation, migration, differentiation, and stability, via welldefined integrin-mediated intracellular signaling pathways $[39,40]$. Endothelial cells form the inner layer of blood vessels and, as such, represent the first layer of resistance to the passage of cells (e.g., inflammatory leukocytes) or substances attempting to pass through the BBB into the cerebral parenchyma. Current evidence now suggests that the molecular basis of the BBB depends on three main factors: (i) inter-endothelial tight junction proteins, (ii) endothelial adhesion to the ECM proteins of the underlying vascular basal lamina, and (iii) the influence of astrocyte end-feet and pericytes [11-14]. Thus far, most attention has focused on the role of tight junction proteins in establishing and maintaining this barrier, while relatively few studies have examined the role of cell adhesion mechanisms in regulating BBB formation and integrity. However, a growing number of studies suggest that ECM-integrin interactions are not just essential for vascular formation and remodeling, but also play an important role in mediating $\mathrm{BBB}$ integrity. While it has been known for some time that laminin imparts an important differentiation and stabilizing influence on endothelial cells [15-19], more recent studies show that it is also required for vascular integrity, as genetic deletion of laminin in astrocytes or pericytes results in defective BBB integrity [20, 21]. In support of this important role for laminin in stabilizing the $\mathrm{BBB}$, a number of studies have shown that loss of the astrocyte laminin receptor, dystroglycan, accompanies vascular leakage at the $\mathrm{BBB}$ and that prevention of this loss ameliorates BBB breakdown [41-43]. Furthermore, several years ago, we showed that pharmacological inhibition of the $\beta 1$ class of integrins leads to increased microvascular permeability in the brain as well as an in vitro endothelial permeability system and that increased vascular permeability correlates with reduced endothelial expression of the tight junction protein claudin-5 [14]. The findings of our current study extend these findings by providing more support for a critical role for laminin and its cellular receptors in maintaining $\mathrm{BBB}$ properties in cerebral blood vessels. To our knowledge, these studies are the first to demonstrate an active upregulation of an adhesive mechanism ( $\alpha 6 \beta 4$ integrin) in response to a potential threat of BBB breakdown.

The unique properties of $\beta 4$ integrin make it well suited to confer enhanced integrity in cerebral blood vessels Amongst cell adhesion molecules, the $\beta 4$ integrin is unique in several ways. First, while most endothelial adhesion molecules (several different $\beta 1$ integrins and dystroglycan) are expressed universally by all types of 
blood vessel, in the normal CNS, $\beta 4$ integrin expression is restricted specifically to arterioles [22], but interestingly during neuroinflammatory conditions, $\beta 4$ integrin is strongly upregulated by all types of blood vessel [23, 24], demonstrating a unique response property of this integrin. Second, the cytoplasmic domain of the $\beta 4$ integrin subunit ( $\sim 1000$ amino acids) is much longer and quite different from the highly conserved cytoplasmic domain other $\beta$ integrin subunits ( 50 amino acids), implying greater potential for unique interactions with cytoskeletal adaptor proteins and intracellular signaling pathways [44]. This idea is supported by the finding that while the cytoplasmic domain of most integrin $\beta$ subunits (e.g., $\beta 1, \beta 3$, and $\beta 5$ ) interacts with the actin cytoskeleton $[37,40]$, the $\beta 4$ integrin cytoplasmic domain interacts specifically with intermediate filament proteins such as vimentin and desmin $[45,46]$. Third, in the epidermis, $\beta 4$ integrin plays an essential role in promoting mechanical stability and barrier integrity, such that mutation or deletion of $\beta 4$ integrin results in skin fragility and blistering in humans and perinatal lethality in $\beta 4$ integrin global $\mathrm{KO}$ mice [25-27]. Based on this collective evidence, we postulate that endothelial cells of the BBB respond to the threat of leukocyte infiltration by increasing $\beta 4$ integrin expression, thus strengthening the transmembrane linkage connecting the basal lamina laminin with the cytoskeletal proteins of endothelial cells, resulting in enhanced BBB integrity.

\section{Does upregulation of endothelial $\beta 4$ integrin hold therapeutic potential?}

Our studies revealed that absence of endothelial $\beta 4$ integrin had no impact on the initial timing of onset of EAE, consistent with a lack of $\beta 4$ integrin expression on microvascular endothelium at this early time point, but as EAE developed, $\beta 4$ integrin was upregulated on vascular endothelium to provide significant protection in the WT mice compared to $\beta 4$-EC-KOs. These findings suggest that factors that enhance endothelial $\beta 4$ integrin expression might afford increased protection against neuroinflammation. Importantly, several studies have shown that statins, cholesterol-lowering drugs that exert potent vasculo-protective and anti-inflammatory effects [47-49], are strong inducers of $\beta 4$ integrin gene expression in endothelial cells. This was first demonstrated in a microarray study of human umbilical vein endothelial cells (HUVECs), in which from a panel of more than 6000 candidate genes, $\beta 4$ integrin stood out as being the gene which showed the greatest (30-fold) increase following treatment with atorvastatin or pitavastatin [50]. Since this time, other studies have confirmed this finding $[51,52]$ and also shown that statins prevent breakdown of cerebrovascular basal lamina proteins in ischemic stroke, consistent with a protective role in preserving BBB integrity [53]. As the beneficial anti-inflammatory effects of statins are well proven [47-49], as well as their ability to suppress EAE progression [54-56], it is tempting to speculate that some of this protection may be in part due to enhanced endothelial $\beta 4$ integrin expression. In subsequent experiments, we will test this hypothesis by treating mice with atorvastatin to stimulate endothelial $\beta 4$ integrin expression on cerebral blood vessels and compare the anti-inflammatory effects of atorvastatin in $\beta 4-E C-K O$ mice and WT littermates. If our prediction is correct, a significant part of the protection conferred by atorvastatin will be lost in $\beta 4$-EC-KO mice. In a more direct approach, we also plan to generate a novel transgenic mouse strain in which $\beta 4$ integrin is specifically over-expressed in endothelial cells and evaluate whether this strain is protected against EAE.

\section{Conclusions}

The goal of this study was to examine the contribution of endothelial $\alpha 6 \beta 4$ integrin to BBB integrity in neuroinflammatory disease. IF analysis showed that endothelial $\beta 4$ integrin expression was markedly upregulated during EAE progression, such that by the acute stage of EAE (day 21), the vast majority of blood vessels expressed $\beta 4$ integrin. In the EAE model, while the $\beta 4$-EC-KO mice showed the same time of disease onset as the WT littermates, they developed significantly worse clinical disease over time, resulting in increased clinical score at the peak of disease and maintained elevated thereafter. Consistent with this, the $\beta 4$-EC-KO mice showed enhanced levels of leukocyte infiltration and BBB breakdown and also displayed increased loss of the endothelial tight junction proteins claudin-5 and ZO-1. Under proinflammatory conditions, primary cultures of $\beta 4 \mathrm{KO}$ BECs also showed increased loss of claudin- 5 expression. Taken together, our data suggest that $\alpha 6 \beta 4$ integrin upregulation is an inducible protective mechanism that stabilizes the BBB during neuroinflammatory conditions.

\section{Abbreviations \\ BBB: Blood-brain barrier; BEC: Brain endothelial cells; CFA: Complete Freund's adjuvant; CNS: Central nervous system; Dual-IF: Dual immunofluorescence; EAE: Experimental autoimmune encephalomyelitis; ECM: Extracellular matrix; FOV: Field of view; HSPG: Heparan sulfate proteoglycan; IFN-y: Interferon gamma; KO: Knockout; MHC: Major histocompatibility complex; MOG: Myelin oligodendrocyte protein; MS: Multiple sclerosis; SEM: Standard error of the mean; SMA: Smooth muscle actin; TNF-a: Tumor necrosis factor-alpha; WT: Wild-type; ZO-1: Zonula occludens-1}

\section{Acknowledgements}

Not applicable

\section{Funding}

This work was supported by a National Multiple Sclerosis Society Post-Doctoral Fellowship to JWW (FG 1879-A-1) and by the NIH RO1 grant NS060770. This is manuscript number 29490 from The Scripps Research Institute.

\section{Availability of data and materials}

The datasets used and/or analyzed during the current study are available from the corresponding author on reasonable request. 


\section{Authors' contributions}

$J W$ generated the $\beta 4-E C-K O$ transgenic mice and together with $A B$, established the EAE studies, analyzed the clinical EAE progression, and performed some of the histological analysis. SKH and RK also performed the histological analysis and contributed to drafting the manuscript. RM conceived of the study, cultured the primary BECs, performed the IF studies of BECs and the flow cytometry, and drafted the manuscript. All authors read and approved the final manuscript.

\section{Ethics approval}

The studies described have been reviewed and approved by The Scripps Research Institute Institutional Animal Care and Use Committee.

\section{Consent for publication}

Not applicable

\section{Competing interests}

The authors declare that they have no competing interests.

\section{Publisher's Note}

Springer Nature remains neutral with regard to jurisdictional claims in published maps and institutional affiliations.

Received: 13 June 2017 Accepted: 24 October 2017

\section{Published online: 09 November 2017}

\section{References}

1. Ffrench-Constant C. Pathogenesis of multiple sclerosis. Lancet. 1994; 343:271-5.

2. Lassmann H. Multiple sclerosis pathology. In: Compston A, editor. McAlpine's multiple sclerosis Churchill Livingstone. 3rd ed; 1998. p. 323-58.

3. Gay D, Esiri M. Blood-brain barrier damage in acute multiple sclerosis plaques. Brain. 1991;114:557-72

4. Kirk J, Plumb J, Mirakhur M, McQuaid S. Tight junction abnormality in multiple sclerosis white matter affects all calibres of vessel and is associated with blood-brain barrier leakage and active demyelination. J Pathol. 2003; 201:319-27.

5. Roscoe WA, Welsh ME, Carter DE, Karlik SJ. VEGF and angiogenesis in acute and chronic MOG (35-55) peptide induced EAE. J Neuroimmunol. 2009;209:6-15.

6. Seabrook TJ, Littlewood-Evans A, Brinkmann V, Pollinger B, Schnell C, Hiestand PC. Angiogenesis is present in experimental autoimmune encephalomyelitis and pro-angiogenic factors are increased in multiple sclerosis lesions. J Neuroinflammation. 2010;7:95.

7. Ballabh P, Braun A, Nedergaard M. The blood-brain barrier: an overview: structure, regulation and clinical implications. Neurobiol Dis. 2004;16:1-13.

8. Brett FM, Mizisin AP, Powell HC, Campbell IL. Evolution of neuropathologic abnormalities associated with blood-brain barrier breakdown in transgenic mice expressing interleukin-6 in astrocytes. J Neuropath Exp Neurol. 1995; 54:766-75.

9. Huber JD, Egleton RD, Davis TP. Molecular physiology and pathophysiology of tight junctions in the blood-brain barrier. Trends Neourosci. 2001;24:719-25.

10. Pardridge WM. Blood-brain barrier drug targetting: the future of brain drug development. Mol Med. 2003;3:90-105.

11. Wolburg H, Lippoldt A. Tight junctions of the blood-brain barrier; development, composition and regulation. Vasc Pharmacol. 2002;38:323-37.

12. Daneman $R$, Zhou $L$, Kebede AA, Barres BA. Pericytes are required for blood-brain barrier integrity during embryogenesis. Nature. 2010;468:562-6.

13. del Zoppo GJ, Milner R. Integrin-matrix interactions in the cerebral microvasculature. Arterioscler Thromb Vasc Biol. 2006:26:1966-75.

14. Osada T, Gu Y-H, Kanazawa M, Tsubota Y, Hawkins BT, Spatz M, Milner R, del Zoppo GJ. Interendothelial claudin-5 expression depends on cerebral endothelial cell-matrix adhesion by $\beta 1$ integrins. J Cereb Blood Flow Metab. 2011;31:1972-85.

15. Davis GE, Camarillo CW. Regulation of endothelial cell morphogenesis by integrins, mechanical forces, and matrix guidance pathways. Exp Cell Res. 1995;216:113-23.

16. Grant DS, Kleinman HK. Regulation of capillary formation by laminin and other components of the extracellular matrix. EXS. 1997;79:317-33.

17. Grant DS, Tashiro K, Segui-Real B, Yamada Y, Martin GR, Kleinman HK. Two different laminin domains mediate the differentiation of human endothelial cells into capillary-like structures in vitro. Cell. 1989;58:933-43.
18. Ingber DE, Folkman J. Mechanochemical switching between growth and differentiation during fibroblast growth factor-stimulated angiogenesis in vitro: role of extracellular matrix. J Cell Biol. 1989;109:317-30.

19. Smyth N, Vatansever HS, Murray P, Meyer M, Frie C, Paulsson M, Edgar D. Absence of basement membranes after targeting the LAMC1 gene results in embryonic lethality due to failure of endoderm differentiation. J Cell Biol. 1999;144:151-60.

20. Chen Z-L, Yao YMM, Norris EH, Kruyer A, Jno-Charles O, Akbarshakh A, Strickland S. Ablation of astrocytic laminin impairs vascular smooth muscle cell function and leads to hemorrhagic stroke. J Cell Biol. 2013;202:381-95.

21. Menezes MJ, McClenahan FK, Leiton CV, Aranmolate A, Shan X, Colognato $H$. The extracellular matrix protein laminin a2 regulates the maturation and function of the blood-brain barrier. J Neurosci. 2014;12:15260-80.

22. Welser-Alves J, Boroujerdi A, Tigges U, Wrabetz L, Feltri ML, Milner R. Endothelial $\beta 4$ integrin is predominantly expressed in arterioles, where it promotes vascular remodeling in hypoxic brain. Arterioscler Thromb Vasc Biol. 2013:33:943-53.

23. Hashimoto T, Meng H, Young WL. Intracranial aneurysms: links between inflammation, hemodynamics and vascular remodeling. Neurol Res. 2006;28:372-80.

24. Milner R, Campbell IL. Increased expression of the beta 4 and alpha 5 integrin subunits in cerebral blood vessels of transgenic mice chronically producing the pro-inflammatory cytokines IL-6 or IFN-alpha in the central nervous system. Mol Cell Neurosci. 2006;33:429-40.

25. Dowling J, Yu Q-C, Fuchs E. $\beta 4$ integrin is required for hemidesmosome formation, cell adhesion and cell survival. J Cell Biol. 1996:134:559-72.

26. Nakano A, Pulkkinen L, Murrell D, Rico J, Lucky AW, Garzon M, Stevens CA Robertson S, Pfendner E, Uitto J. Epidermolysis bullosa with congenital pyloric atresia: novel mutations in the beta 4 integrin gene (ITGB4) and genotype/phenotype correlations. Pedatr Res. 2001:49:618-26.

27. van der Neut R, Krimpenfort P, Calafat J, Niessen CM, Sonnenberg A. Epithelial detachment due to absence of hemidesmosomes in integrin beta 4 null mice. Nat Genet. 1996;13:366-9.

28. Kisanuki YY, Hammer RE, Miyazaki J, Williams SC, Richardson JA, Yanagisawa M. Tie2-Cre transgenic mice: a new model for endothelial cell-lineage analysis in vivo. Dev Biol. 2001:230:230-42.

29. Tronche F, Kellendonk C, Kretz O, Gass P, Anlag K, Orban PC, Bock R, Klein R, Schutz G. Disruption of the glucocorticoid receptor gene in the nervous system results in reduced anxiety. Nat Genet. 1999;23:99-103.

30. Nodari A, Previtali SC, Dati G, Occhi S, Court FA, Colombelli C, Zambroni D, Dina G, Del Carro U, Campbell KP, et al. a6ß4 integrin and dystroglycan cooperate to stablize the myelin sheath. J Neurosci. 2008;28:6714-9.

31. Crocker SJ, Whitmire JK, Frausto RF, Chertboonmuang P, Soloway PD, Whitton JL, Campbell IL. Persistent macrophage/microglial activation and myelin disruption after experimental autoimmune encephalomyelitis in tissue inhibitor of metalloproteinase-1-deficient mice. Am J Pathol. 2006;169: 2104-16.

32. Milner R, Crocker SJ, Hung S, Wang X, Frausto RF, Del Zoppo GJ. Fibronectin- and vitronectin-induced microglial activation and matrix metalloproteinase- 9 expression is mediated by integrins a5ß1 and avß5. J Immunol. 2007;178:8158-67.

33. Milner R, Campbell IL. Developmental regulation of $\beta 1$ integrins during angiogenesis in the central nervous system. Mol Cell Neurosci. 2002;20:616-26.

34. Milner R, Hung S, Wang X, Berg G, Spatz M, del Zoppo G. Responses of endothelial cell and astrocyte matrix-integrin receptors to ischemia mimic those observed in the neurovascular unit. Stroke. 2008;39:191-7.

35. Tigges U, Welser-Alves JV, Boroujerdi A, Milner R. A novel and simple method for culturing pericytes from mouse brain. Microvasc Res. 2012;84:74-80.

36. Milner R, Campbell IL. The extracellular matrix and cytokines regulate microglial integrin expression and activation. J Immunol. 2003;170:3850-8.

37. Hemler ME. Integrins. In: Kreis T, Vale R, editors. In guidebook to the extracellular matrix, anchor and adhesion proteins. New York: Oxford University Press; 1999. p. 196-212.

38. Boroujerdi A, Welser-Alves J, Milner R. Extensive vascular remodeling in the spinal cord of pre-symptomatic experimental autoimmune encephalomyelitis mice; increased vessel expression of fibronectin and the a5 31 integrin. Exp Neurol. 2013;250:43-51.

39. Hynes RO. Genetic analyses of cell-matrix interactions in development. Curr Opin Genet Dev. 1994;4:569-74.

40. Hynes RO. Integrins: bidirectional allosteric signaling machines. Cell. 2002; 110:673-87. 
41. Agrawal S, Anderson P, Durbeej M, van Rooijen N, Ivars F, Opdenakker G, Sorokin LM. Dystroglycan is selectively cleaved at the parenchymal basement membrane at sites of leukocyte extravasation in experimental autoimmune encephalomyelitis. J Exp Med. 2006;203:1007-19.

42. Milner R, Hung S, Wang X, Spatz M, del Zoppo G. The rapid decrease in astrocyte-associated dystroglycan expression by focal cerebral ischemia is protease-dependent. J Cereb Blood Flow Metab. 2008;28:812-23.

43. Wolburg-Burcholz K, Mack AF, Steiner E, Pfeiffer F, Engelhardt B, Wolburg H. Loss of astrocyte polarity marks blood-brain barrier impairment during experimental autoimmune encephalomyelitis. Acta Neuropathol. 2006;118:219-33.

44. Feltri ML, Arona M, Scherer SS, Wrabetz L. Cloning and sequence of the CDNA encoding the beta 4 integrin subunit in rat peripheral nerve. Gene. 1997;186:299-304.

45. Homan SM, Martinez R, Benware A, LaFlamme SE. Regulation of the association of alpha 6 beta 4 with vimentin intermediate filaments in endothelial cells. Exp Cell Res. 2002;281:107-14.

46. Litjens SHM, de Pereda JM, Sonnenberg A. Current insights into the formation and breakdown of hemidesmosomes. Trends Cell Biol. 2006; 16:376-83.

47. Ifergan I, Wosik K, Cayrol R, Kebir H, Auger C, Bernard M, Bouthillier A, Moumdijian R, Duquette P, Prat A. Statins reduce human blood-brain barrier permeability and restrict leukocyte migration: relevance to multiple sclerosis. Ann Neurol. 2006;60:45-55.

48. Jacobson JR, Barnard JW, Grigoryev DN, Ma SF, Tuder RM, Garcia JGN. Simvastatin attenuates vascular leak and inflammation in murine lung injury. Am J Physiol Lung Cell Mol Physiol. 2005;288:L1026-32.

49. Moonis M, Kane K, Schwiderski U, Sandage BW, Fisher M. HMG-CoA reductase inhibitors improve acute ischemic stroke outcome. Stroke. 2005; 36:1298-300.

50. Morikawa S, Takabe W, Mataki C, Wada Y, Izumi A, Saito Y, Hamakubo T, Kodama T. Global analysis of RNA expression profile in human vascular cells treated with statins. J Atheroscl Thromb. 2004;11:62-72.

51. Feng C, Ye C, Liu X, Ma X, Li M. b4 integrin is involved in statin-induced endothelial cell death. Biochem Biophys Res Commun. 2004;323:858-64.

52. Jacobson JR, Dudek SM, Birukov KG, Ye SQ, Grigoyev DN, Girgis RE, Garcia JGN. Cytoskeletal activation and altered gene expression in endothelial barrier regulation by simvastatin. Am J Respir Cell Mol Biol. 2004;30:662-70.

53. Trinkl A, Vosko MR, Wunderlich N, Dichgans M, Hamann GF. Pravastatin reduces microvascular basal lamina damage following focal cerebral ischemia and reperfusion. Eur J Neurosci. 2006;24:520-6.

54. Luccarini IL, Ballerini C, Biagioli T, Biamonte F, Bellucci A, Rosi MC, Grossi C, Massacesi L, Casamenti F. Combined treatment with atorvastatin and minocycline suppresses severity of EAE. Exp Neurol. 2008;211:214-26.

55. Stanislaus R, Pahan K, Singh AK, Singh I. Amelioration of experimental allergic encephalomyelitis in Lewis rats by lovastatin. Neurosci Lett. 1999; 269:71-4.

56. Youssef S, Stuve O, Patarrayo JC, Ruiz PJ, Radosevich JL, Hur EM, Bravo M, Mitchell DJ, Sobel RA, Steinman L, Zamvil SS. The HMG-CoA reductase inhibitor, atorvastatin, promotes a Th2 bias and reverses paralysis in central nervous system autoimmune disease. Nature. 2002;420:78-84.

\section{Submit your next manuscript to BioMed Central and we will help you at every step:}

- We accept pre-submission inquiries

- Our selector tool helps you to find the most relevant journal

- We provide round the clock customer support

- Convenient online submission

- Thorough peer review

- Inclusion in PubMed and all major indexing services

- Maximum visibility for your research

Submit your manuscript at www.biomedcentral.com/submit 